\title{
TRANSMISSION EQUIPMENT TESTING BASED ON INTERNET OF THINGS
}

\author{
Qing $\mathrm{Lv}^{1}$, Chao Fu ${ }^{2}$ \\ ${ }^{1}$ Hebei Normal University, Hebei, 050024, China \\ ${ }^{2}$ Corresponding author: Chao $\mathrm{Fu}$ \\ Email: fuchao hbnu@163.com
}

\begin{abstract}
In order to reduce the temporary failure of transmission equipment, intelligent information fusion technology was introduced. The intelligent processing of information in the Internet of things was implemented. Then, the online detection information and inspection information in the Internet of things were applied. The advantages and disadvantages of various information fusion methods were compared. The integration method of fuzzy evidence theory was introduced. Based on the fuzzy theory and the evidence theory, the integrated information fusion fault diagnosis model was established. According to the fusion model, the fault information preprocessing was realized by means of membership function and bipolar ratio method. In the local fusion diagnosis based on fuzzy theory, AHP was used to construct the fuzzy transformation matrix. In the decision fusion diagnosis based on evidence theory, the integrated local fusion results were integrated to realize the decision fusion of the fault information. Accurate diagnosis results were obtained. Finally, the example of power transformer was analysed. The results showed that the diagnostic results of this method were accurate. Therefore, this method can effectively improve the accuracy and reliability of diagnosis, and reduce the uncertainty of diagnosis. It provides an effective way and method for the research of fault diagnosis of transmission and transformation equipment.
\end{abstract}

Keywords: Internet of things; transmission equipment; fault detection

\section{Introduction}

Electricity is the economic lifeline of the country. It is not only one of the most important basic industries supporting the national economy, but also a basic part of the national energy security [1]. Generally, the power system is a system of electricity production, transmission and consumption. It consists of electricity generation, transmission, substation, distribution and electricity [2]. Among them, transmission and substation play an extremely important role in the power system, and is also an important part of the construction of smart grid [3].

Transmission and transformation equipment, especially large high-voltage equipment, transmission lines, etc., will cause huge economic losses and adverse social impacts in the event of a sudden failure [4]. Therefore, the reliability of power transmission and transformation equipment is the basis of power system security, stability and economic operation [5].

In order to improve the reliability of power transmission and transformation equipment, the quality of the equipment has been improved, the high-quality materials have been selected, the advanced technology has been utilized and the design has been optimized, so that no failure occurs in the working life $[6,7]$. However, this will lead to increased manufacturing costs. With the running time going on, the equipment always gets old and the equipment failure rate will get higher and higher [8].

Therefore, the necessary inspection and diagnosis of the equipment must be carried out. The hidden dangers of the equipment are eliminated in time. The maintenance equipment is a very important work content of the power enterprise $[9,10]$.

In order to improve the accuracy and real-time performance of the fault diagnosis of the transmission and transformation equipment, the Internet of things technology is introduced into the fault diagnosis.

With the advantages of information perception, acquisition and communication, the real-time performance of fault diagnosis and the overall status of state information are guaranteed. By means of information fusion technology, the state information is synthetically analysed and fused by using intelligent algorithms, to get more accurate diagnosis results. 


\section{Methodology}

\subsection{Online information detection}

Online detection information: it can reflect the actual conditions of the equipment. When the failure just happened, the fault was detected. It is the most important basis for equipment fault diagnosis. Therefore, the electric power sector is using online detection device to replace the periodic electrical test, to get the status information of the device. The online detection device is also increasing. Power transmission and transformation equipment covers a wide range. There are many kinds of equipment.

The environmental information that is directly related to them is also extremely rich. Therefore, in the network of transmission and transformation equipment, the sensors need not only a large number of sensors, but also many types of sensors. Usually, these multiple types of sensors form a sensor network to realize the perception and acquisition of equipment state information and environmental information. Of course, according to the importance of state information, the sensor sets a fixed time interval of information acquisition, and actively perceiving and collecting the state information of the equipment. The information is transmitted to the storage system of the information platform of the transmission and transformation equipment, according to a certain data format.

By deploying sensor network, the status information of transmission and transformation equipment can be obtained by on-line detection system. For example, the online detection information provided by the Internet of things for power transformers includes iron core grounding current, dissolved gas content in oil, micro water content in oil, dielectric loss coefficient, and partial discharge capacity, DC resistance imbalance coefficient of winding and winding deformation. This information is all the common signs of fault diagnosis. In particular, some scholars and organizations have done a great deal of research on the dissolved gases in oil. The on-line detection information is closely related to the breakdown of the high-voltage circuit breaker, such as the time of closing and closing, the voltage and current of the closing coil, the speed of the moving contact and the stroke.

Defect information: the defect information of the equipment includes many aspects. Family defects have an important impact on the equipment. Family defects are equipment defects that are identified by the common factors such as material, design and process. If such defects occur, other devices with the same material, design and technology, regardless of whether they can detect similar defects at present, are called family defect devices before they are eliminated. Family defect information can diagnose all kinds of abnormal states or fault states in time and correctly, prevent or eliminate faults, and guide the operation of equipment to improve the reliability, safety and effectiveness of equipment operation. The loss was reduced to the lowest level.

Defect information records defect discovery and processing time, defect nature, defect type, defect number, defect location, defect cause, severity, temporary measure and left-over problem. As shown in Figure 1, generally, defect information is first encapsulated by the Internet of things common information model (CIM) or its extended model, and the information is connected to the information platform of the Internet of things through a unified data interface. After data extraction, conversion, loading and cleaning, the storage of defect information is completed.

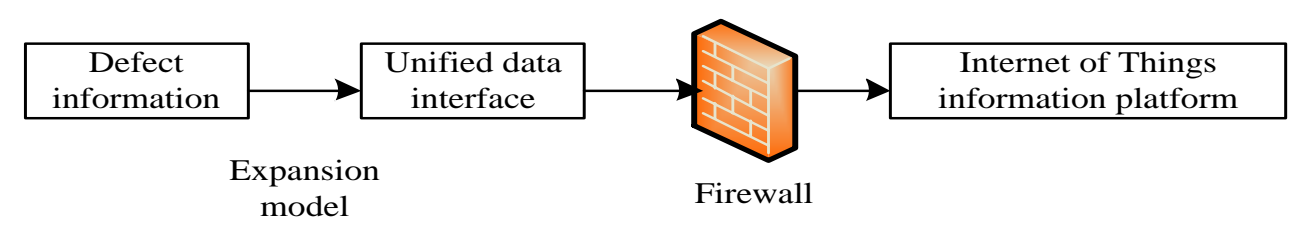

Figure 1: Defect information access to the information platform of the Internet of things

Inspection: through the sound, vibration, smell, colour and other human feelings, inspection determines the operating state of the equipment. According to the changes of the observed phenomena, the location and degree of the failure are analyzed. It is an important aspect of fault detection and diagnosis. Human feeling is adulterated with human subjective factors. The degree of feeling is difficult to quantify, and it can only be approximated by a language. However, these signs and failures have a certain causal relationship.

For example, the abnormal sound of the transformer can be caused by the loosening of the screw of the iron core or the internal clamp, or the overload of the transformer. Therefore, this detection method is still widely used in electric power enterprises. The traditional inspection adopts the way of paper recording, which is obviously unable to meet the requirements of real-time diagnosis. The development of Internet of things and RFID technology can provide paperless electronic records for intelligent patrol inspection, and transmit real-time and reliable inspection information in a certain data format to the information platform of the Internet of things. For example, when the power transformer is inspected 
and checked, the portable reader will record the results of various inspection items one by one, and send the information to the information platform accurately and in real time.

Preventive test information: it is an important source of information for a simple and effective fault diagnosis. Through a variety of effective tests, reliable and accurate test results are the basic prerequisites for the accurate diagnosis of the state of the transmission and transformation equipment.

The test information obtained by the preventive test is the main basis for the equipment maintenance, and it also plays an active role in the safe operation of the electric power equipment. Preventive tests include non-destructive tests and destructive tests. A test at a lower voltage (below or close to the rated voltage) is called a non-destructive test. Because of the low working voltage applied to this type of test, the insulation performance of the equipment will not be damaged. The purpose is to judge the state of insulation and to find the possible deterioration in time. Destructive tests are carried out at higher than rated voltage or working voltage. The test voltage is added to the insulation of the equipment in the test, and the tolerance to this voltage is tested. Therefore, it is called pressure test. It is mainly used to measure the AC voltage and DC voltage of the electric power equipment. Because of the higher voltage added to this kind of test, the test is more direct and strict.

There may be some damage to the power equipment during the test.

For example, 32 transformer tests, 12 transformer oil tests and 5 transformer casing tests are included in the preventive test of the transformer. Regular test items include infrared thermography, dissolved gas in oil analysis, winding resistance, insulating oil casing test, routine test, core insulation resistance, winding insulation resistance, load switch, check the decomposition temperature measuring device, check the winding insulation dielectric loss factor, temperature measuring device, check the gas relay check, cooling device inspection and pressure release device check.

The way that the pre-experiment information is connected to the information platform of the Internet of things is the same as the defect information and the operating condition information.

\subsection{The level of information fusion}

The information in the Internet of things includes many aspects. In the process of information fusion, it is necessary to integrate the multi-source information one by one. The fusion results are obtained and integrated, which involves the hierarchical structure of the fusion. Generally, information fusion can be divided into three layers: data level fusion, feature level fusion and decision level fusion.
Data level fusion: data level fusion is the lowest level of fusion. It directly integrates the original data without pre-processing, and then extracts and determines the decision based on the fusion results. Its fusion process is shown in Figure 2. The main advantage is that during the fusion process, the amount of data lost is the least, which can keep as much raw information as possible and provide some fine information with high accuracy. The limitation is that the amount of information that needs to be processed is large, so the processing time is longer, the real time is the worst, and the cost is the highest. Because of the instability of the original information, the fusion result is inaccurate, which requires a strong error correction processing ability in the data fusion. Since the original data is not pre-processed, it is directly related, so the multi-source information in the Internet of things is required to have the same data type.

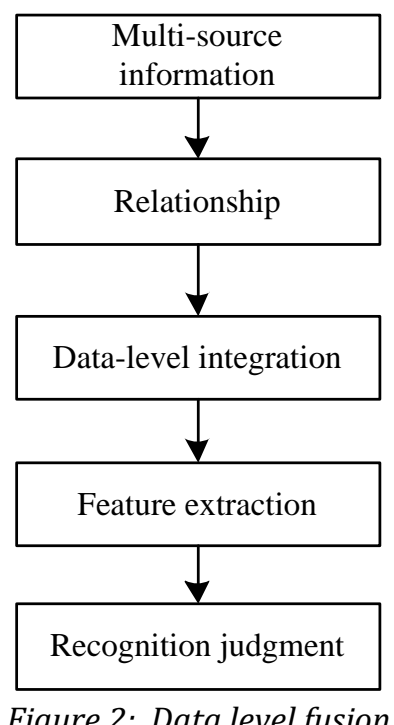

Feature level fusion: the fusion of the feature level is the fusion of the middle level. The fusion process is shown in Figure 3. First, the original information from all aspects is pre-processed and the features are extracted. Then, the feature information is related, analysed and processed. The final fusion results are obtained. The main advantage is that the information compression is realized and the amount of information is reduced, which is beneficial to the real-time processing of the feature information. The various features extracted are related to the final identification and judgment results. Therefore, the result of the feature level fusion can provide a variety of feature information for the decision analysis to the maximum extent. The limitation is that some useful information will no longer be retained because of the compression of information and other reasons. In this way, the performance of the fusion is slightly reduced. 


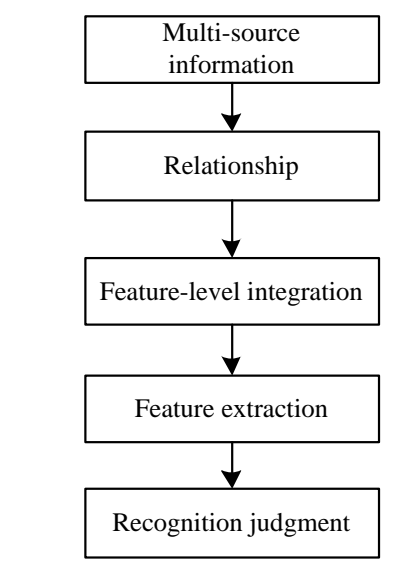

Figure 3: Feature level fusion

Decision level fusion: decision level fusion is the highest level of integration. It needs to start from the needs of specific decision problems, make full use of all kinds of feature information extracted from feature level fusion, and adopt decision fusion algorithm and follow certain criteria to achieve final decision. Decision level fusion is directly aimed at specific decision goals. The results of the fusion will directly affect the accuracy of recognition and judgment. The process of fusion is shown in Figure 4.

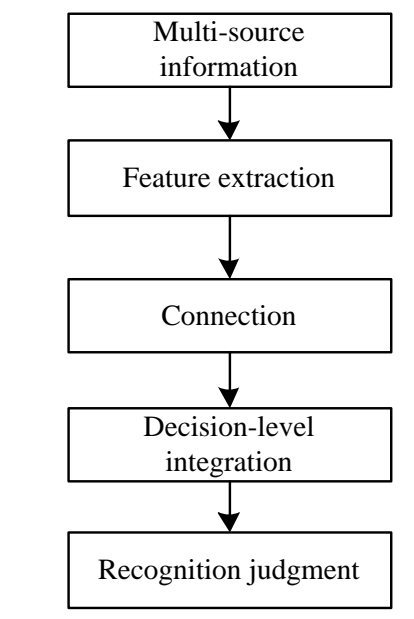

Figure 4: Decision level fusion

The main advantage is that the flexibility is very high. It is not very demanding for the data structure of the information, which can be isomorphic or isomeric. When certain information in the multisource information is inaccurate, the accurate results can be obtained by proper fusion. Therefore, it has a strong fault tolerance. The limitation lies in the decision - level fusion that first needs to be preprocessed to each of the original information to obtain the results of their own judgment. All preprocessing costs are high.

\subsection{The model and steps of information fusion}

The information of the Internet of things comes from different aspects. According to the level of information fusion, a multi-level fault diagnosis information fusion model is established, as shown in Figure 5.

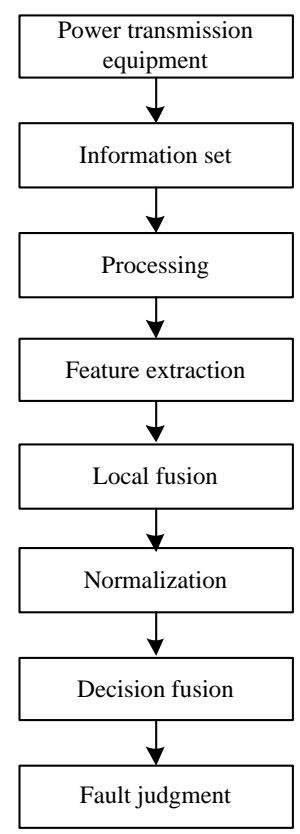

Figure 5: Decision fusion and diagnosis

According to the information fusion model of fault diagnosis, the fault diagnosis of transmission and transformation equipment has the following steps.

The determination of fault sets and symptom sets: fault sets can be determined according to the previous fault conditions of the same type of equipment. The omen information comes from the online detection, inspection, pre-experiment, defect and other aspects of the Internet of things. Therefore, the symptom set is divided into on-line detection of symptom information subsets, symptom information subset, pre-inspection information, experiment empty defect information subset. In some cases, the subset of the omen information can be further subdivided.

Pre-processing and feature extraction of symptom information: in order to facilitate information fusion, after identifying the fault set and symptom set, the symptom information in the Internet of things needs to be dealt with preliminarily. The useful information related to the fault is extracted and analyzed. Generally, the methods used in pre-processing and feature extraction include Fourier transform, wavelet transform and fuzzy technique.

Local fusion diagnosis: online detection information, inspection information, pre-test information and defect information after pretreatment and feature extraction are respectively regarded as their local fusion inputs. With the help of fuzzy theory, artificial neural network, rough set theory, expert system and other intelligent 
algorithms, these characteristics are fused. The mapping from the subset of the omen to the fault set is implemented. The results of local fusion are obtained, that is, the possibility of each fault.

Decision fusion diagnosis: using evidence theory, Bayesian theory and other decision fusion methods, the normalized local fusion results are fused. The final diagnosis results are obtained. Before decision fusion, the reliability coefficient of each local fusion sub network must be determined.

\subsection{Fault diagnosis system architecture of transmission and transformation equipment based on Internet of things}

As with the IoT architecture for smart grid applications, the IoT-based fault diagnosis system architecture for transmission and distribution equipment is also divided into three layers: perception layer, communication layer and application layer, as shown in Figure 6.

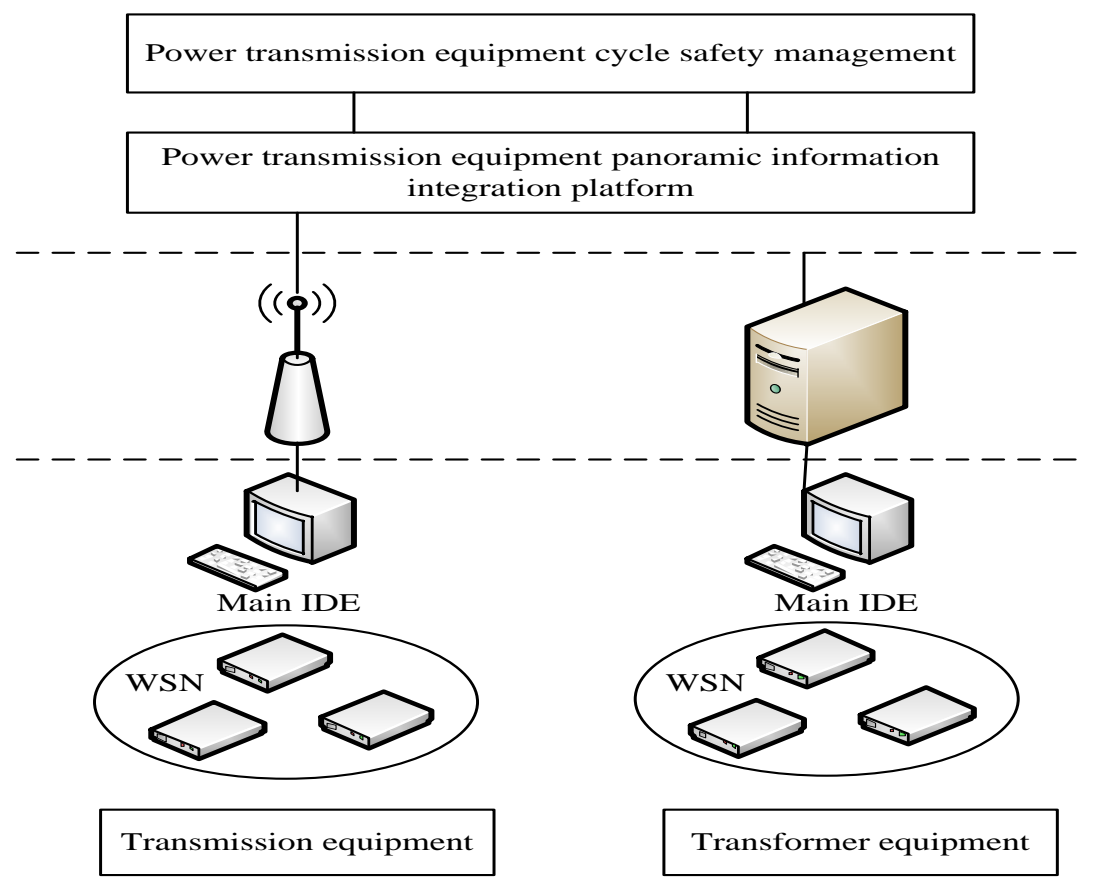

Figure 6: Fault diagnosis system simulation of transmission and transformation equipment based on Internet of things

Through the sensor network and the RFID device, the sensing layer has an effective perception of the transmission and transformation equipment. The online detection information, identification information, attribute information and environmental information of the equipment are collected. The main technologies to support the perceptual layer are RFID, WSN, and GPS and so on. The information aware device of the sensing layer mainly includes many types of smart sensors, detection of IED Intelligent Electric Device, intelligent electronic devices, RFID tags and readers. Among them, the detection master IED not only has the function of the aggregation node in the wireless sensor network, but also can realize the preliminary processing of the online detection information.

In power transmission equipment, wireless sensors such as ice coating, sag, galloping, pulling and so on is deployed on transmission lines to detect and collect on-line detection information of transmission equipment in real time. In the tower, the camera, the anti-theft bolt, the tilt angle sensor and other information sensing equipment are installed to realize the real-time detection and theft protection of the tower. In addition, by reading the RFID tag information, the reader in the main IED is detected to obtain the property of the device and the inspection information, and transmit it to the detection master IED. The initial processing of information has been completed.

\section{Results and discussion}

\subsection{Decision fusion and diagnosis of fault information}

Using the fuzzy theory, the local fusion of on-line detection information and inspection information is realized. According to the rules of fuzzy diagnosis, the results of local diagnosis are obtained. However, there may be inconsistencies in the results of local fusion. How to obtain consistent diagnostic results through effective methods involves decision fusion and diagnosis of fault information.

On the basis of the integration of local fusion results, decision fusion and decision fusion are used 
to get the final decision fusion results. According to a certain criterion, the type of fault is judged, as shown in Figure 7. In this chapter, the decision fusion method is used to synthesize the results of each fuzzy local diagnosis, and to obtain the consistent diagnosis results. There are many methods of decision fusion. Evidence theory is one of the most commonly used and most effective methods.

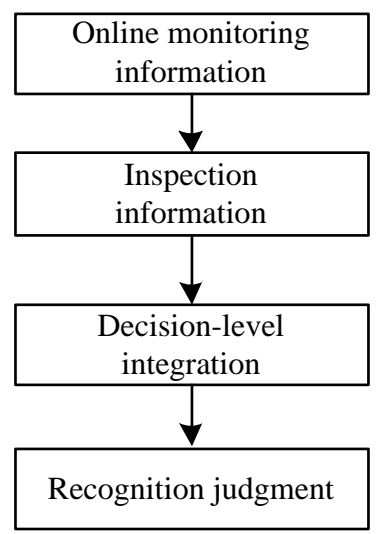

Figure 7 Decision fusion and diagnosis

Evidence theory is one of the main reasoning methods commonly used in information fusion, which is often used to solve the problem of uncertainty.
The theory of evidence is first proposed by Dempster, and then further perfected by Shafer. Therefore, it is called D-S evidence theory, which is a set of mathematical theories about evidence and reasoning. The fusion and diagnosis of fault information decision based on evidence theory is a comprehensive consideration of the results of each local fusion. The results of each local fusion are used as a separate body of evidence. Through the law of evidence synthesis, each body of evidence is synthesized by a new body of evidence. Finally, under certain rules of decision and diagnosis, the type of fault in the equipment is judged.

\subsection{Case study}

A substation 240MVA, 220k V transformer, the model is SFPSZ1-240000/220. Other parts of the online detection information are shown in Table 1 and Table 2. When inspecting, the appearance check found that the transformer has a slight leakage of oil. The main problems of the accessories are: the heating of the winding is detected. The oil temperature is close to 100 degrees. There is obvious noise and vibration when the tap switch operates, and the false oil level is caused by the poor expiratory.

Table 1 Online detection information

\begin{tabular}{|c|c|c|c|c|c|}
\hline $\begin{array}{c}\text { Online } \\
\text { detection } \\
\text { information }\end{array}$ & $\begin{array}{c}\text { Winding DC } \\
\text { resistance } \\
\text { unbalance factor } \\
/ \%\end{array}$ & $\begin{array}{c}\text { Partial } \\
\text { discharge } \\
\text { capacity / pC }\end{array}$ & $\begin{array}{c}\text { Winding loss } \\
/ \%\end{array}$ & $\begin{array}{c}\text { Core ground } \\
\text { current / mA }\end{array}$ & $\begin{array}{l}\text { Oil micro- } \\
\text { water / mg / } \\
\mathrm{L}\end{array}$ \\
\hline Value & 0.55 & 800 & 0.59 & 37.4 & 21 \\
\hline
\end{tabular}

Table 2 Dissolved gas content in oil

\begin{tabular}{|c|c|c|c|c|c|c|c|}
\hline $\begin{array}{c}\text { Dissolved } \\
\text { gas in oil }\end{array}$ & $\mathrm{CO}$ & $\mathrm{CO}_{2}$ & $\mathrm{H}_{2}$ & $\mathrm{CH}_{4}$ & $\mathrm{C}_{2} \mathrm{H}_{2}$ & $\mathrm{C}_{2} \mathrm{H}_{4}$ & $\mathrm{C}_{2} \mathrm{H}_{6}$ \\
\hline $\begin{array}{c}\text { Content } \\
\mathrm{uL} / \mathrm{L}\end{array}$ & 197 & 711 & 92.1 & 28 & 5.72 & 48 & 9.1 \\
\hline
\end{tabular}

The determination of fault sets and symptom sets: according to the large number of historical fault data of the power transformer and the opinions of some transformer diagnostics experts, the fault set $\mathrm{F}$ is determined as: the local short circuit of the core and the multi-point earthling F1, the insulation aging F2, the overheating of the magnetic shielding discharge or the magnetic leakage heating F3, insulation damp F4, lead failure and tap changer accident F5, suspension discharge F6, discharge F7, winding deformation and inter-turn short circuit F8. The elements of the set of signs need to be determined both in terms of online inspection and inspection. In line detection, according to Table 1, the local discharge, the water in oil and the dissolved gas in the oil are used as the sign information. In the field of inspection, according to Table 2, the sound, temperature, and oil level anomalies are used as sign information.

Pre-processing of omen information: online detection information, for local electric quantity, oil in micro water, the membership degree is $0.83,0.1$ respectively. The gas is dissolved in the oil, and the three ratios are coded to 012 by calculation. According to the improved three ratio method, it is judged as arc and overheating, and the content of C2H2 exceeds the standard. The degree of membership is 0.86 . The pre-processing results of the inspection information, such as Table 3 and Figure 8, are obtained by using the two-pole proportion method. 
Table 3 Pre-processing results of inspection information

\begin{tabular}{|c|c|c|}
\hline Abnormal sound & Temperature anomaly & Anomaly of oil level \\
\hline Obvious & Obvious & Change \\
\hline 4 & 4 & 3 \\
\hline
\end{tabular}

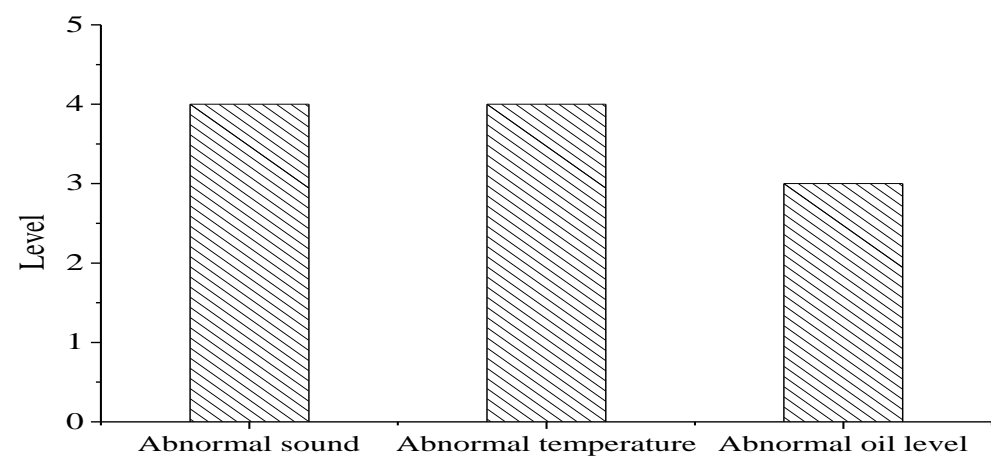

Figure 8 Comparison of the results of inspection information sub item

Decision fusion diagnosis based on evidence theory: first, the framework of identification is constructed. The first 3 diagnostic results were F6, F7, and F8 through two local fusion sub networks. Therefore, the identification framework $F$ of the evidence theory is determined as the floating discharge F6, the screen discharge F7, the winding deformation and inter-turn short circuit F8.

According to a comprehensive diagnosis of the use of online detection data and inspection data of the actual effect of network diagnosis in the power transmission and transformation equipment fault diagnosis and analysis of the existing literature experience, online detection and diagnosis of network reliability coefficient of the manual inspection and diagnosis network is respectively 0.9 , 0.8 . The selection method of science needs to be further studied in the future.
By local fusion, the input of decision fusion is obtained by using the application formula, that is, the basic probability distribution of the propositions on the recognition framework, as shown in Table 4. Using the synthetic rules of the evidence, the distribution of the final reliability distribution for each fault is obtained, as shown in Table 5. Obviously, F8 has the largest basic trust distribution value and is greater than the threshold value of 0.4 . The difference of the reliability function with other fault types is more than 0.2 of the min value. The value of the uncertainty reliability function after synthesis is less than the threshold value of 0.1 . According to the decision and decision rules, the final diagnosis results are the winding deformation and the interturn short circuit F8. This is in accordance with the actual inspection result of the hoisting cover. Figure 9 is a probability comparison of each body of evidence.

Table 4 Basic probability distribution of each evidence body

\begin{tabular}{|c|c|c|c|c|}
\hline Evidence & F6 & F7 & F8 & Diagnostic results \\
\hline $\begin{array}{c}\text { Online detection } \\
\text { information }\end{array}$ & 0.2831 & 0.2237 & 0.3951 & Unknown \\
\hline $\begin{array}{c}\text { Inspection } \\
\text { information }\end{array}$ & 0.2094 & 0.2232 & 0.3674 & Unknown \\
\hline
\end{tabular}

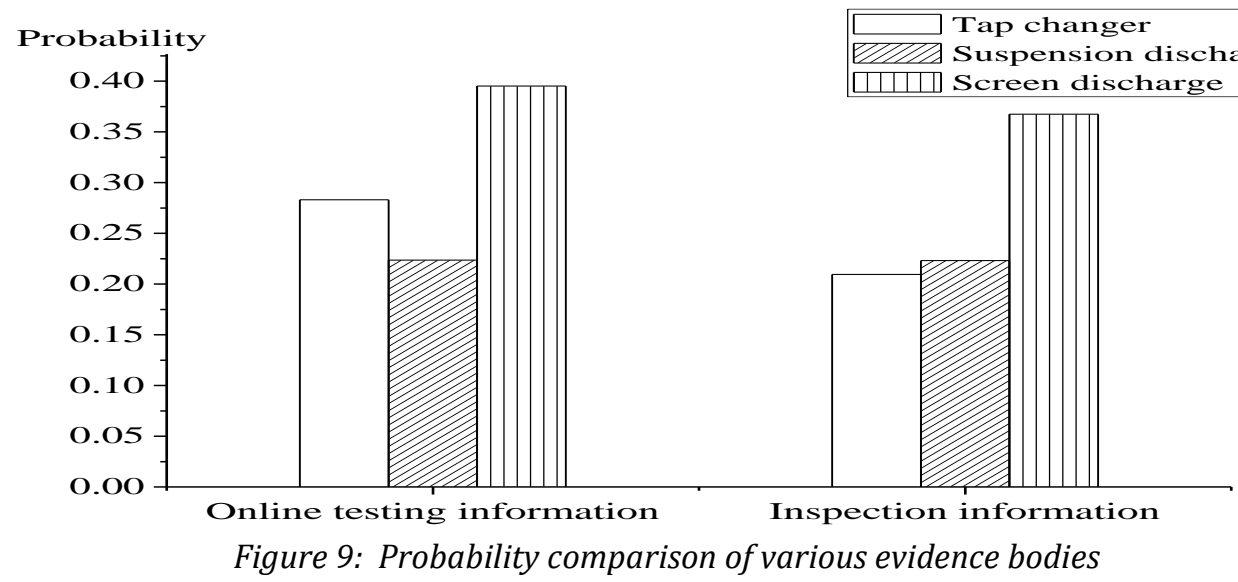


Table 5 The basic trust degree allocation and diagnosis result of decision fusion

\begin{tabular}{|c|c|c|c|c|}
\hline Evidence & F6 & F7 & F8 & Diagnostic results \\
\hline $\begin{array}{c}\text { Online detection information \& } \\
\text { inspection information }\end{array}$ & 0.2567 & 0.2196 & 0.4896 & F8 \\
\hline
\end{tabular}

As can be seen from Table 4 and Table 5, compared with the results of local fusion, the basic credibility of decision fusion is obviously improved and the uncertainty is obviously reduced. This shows that when using fault symptom information of unilateral (online detection information or inspection information) to diagnose and identify faults, the reliability is relatively low and the uncertainty is relatively high. Sometimes, it may not be able to accurately determine the type of fault. Using multiple fault symptoms information, the diagnostic results have been significantly improved. At the same time, the basic credibility of the decision fusion is obviously improved. The uncertainty is greatly reduced. It is beneficial to the more accurate diagnosis and decision of the fault.

\section{Conclusion}

The technology of Internet of things is introduced to the fault diagnosis of transmission and transformation equipment. The Internet of things has powerful and comprehensive information perception and acquisition ability, reliable communication network, and intelligent information processing methods. It can provide comprehensive and real-time state information and information processing for fault diagnosis. The application of some key technologies in the power system in the Internet of things is discussed and summarized. The application of the Internet of things in the smart grid is discussed. A fault diagnosis system architecture for transmission and transformation equipment based on the Internet of things is proposed. Through example analysis, compared with unilateral symptom information (online detection information or patrol information), the comprehensive symptom information provided by Internet of things can effectively improve the accuracy and reliability of diagnosis. The uncertainty of diagnosis was reduced. It provides an effective way and method for the research of fault diagnosis of transmission and transformation equipment.

\section{Acknowledgement}

This work was supported by Hebei Province Education Office Foundation (QN201 5030,QN2016100);

Hebei Normal University Youth Foundation (L2015Q 09,L2015Q12);Technology Foundation for Selected 0 verseas Chinese Scholar of Hebei province (C201500 5016);
Doctoral foundation of Hebei Normal University(L20 17B20)

\section{References}

[1] Lo Y L, Chuang Y H (2017). A High-Efficiency CMOS Rectifier with Wide Harvesting Range and Wide Band Based on MPPT Technique for LowPower IoT System Applications. Circuits Systems \& Signal Processing, 36(12), 5019-5040.

[2] Liu X, Sánchez-Sinencio E (2015). A Highly Efficient Ultralow Photovoltaic Power Harvesting System With MPPT for Internet of Things Smart Nodes. IEEE Transactions on Very Large Scale Integration Systems, 23(12), 3065-3075.

[3] [3]Zeng M, Wang S (2016). Fuzzy Comprehensive Evaluation Algorithm for Power Information System Security Level Based on the Internet of Things. International Journal of Online Engineering, 12(5), 17.

[4] Burdett A (2015). Ultra-Low-Power Wireless Systems: Energy-Efficient Radios for the Internet of Things. IEEE Solid-State Circuits Magazine, $7(2), 18-28$.

[5] Hahm O, Baccelli E, Petersen H, et al (2016). Operating Systems for Low-End Devices in the Internet of Things: A Survey. IEEE Internet of Things Journal, 3(5), 720-734.

[6] Wang Y, Liu Y, Wang C, et al (2016). Storage-Less and Converter-Less Photovoltaic Energy Harvesting With Maximum Power Point Tracking for Internet of Things. IEEE Transactions on Computer-Aided Design of Integrated Circuits and Systems, 35(2), 173-186.

[7] Zhu L, Xu J, Yan L (2016). Research on congestion elimination method of circuit overload and transmission congestion in the internet of things. Multimedia Tools \& Applications, 76(17), 1-20.

[8] Augustin A, Yi J, Clausen T, et al (2016). A Study of LoRa: Long Range \& Low Power Networks for the Internet of Things. Sensors, 16(9), 1466.

[9] Cacciapuoti A S, Caleffi M (2017). Receiver Design for a Bionic Nervous System: Modeling the Dendritic Processing Power. IEEE Internet of Things Journal, 3(1), 27-37.

[10] Singh J, Pasquier T, Bacon J, et al (2017). Twenty Security Considerations for CloudSupported Internet of Things. IEEE Internet of Things Journal, 3(3), 269-284. 